\title{
FUNCTORIAL CONSTRUCTION OF LE BARZ'S TRIANGLE SPACE WITH APPLICATIONS
}

\author{
SEAN KEEL
}

\begin{abstract}
We give a new functorial construction of the space of triangles introduced by Le Barz. This description is used to exhibit the space as a composition of smooth blowups, to obtain a space of unordered triangles, and to study how the space varies in a family.
\end{abstract}

\section{INTRODUCTION}

In [L] Le Barz introduces a smooth variety parameterizing triangles in a given smooth variety. In this paper we give an alternative functorial description of this space, based on a suggestion of Bill Fulton. Le Barz considers for a variety $V$ the space $\widehat{H}$ which is the closure in

$$
\operatorname{Hilb}_{1}(V)^{\times 3} \times \operatorname{Hilb}_{2}(V)^{\times 3} \times \operatorname{Hilb}_{3}(V)
$$

(throughout the introduction we will refer to the above product as HILB) of the locus of triangles with three distinct points. These "honest triangles" are parameterized by

$$
V \times V \times V \backslash \Delta
$$

which one embeds (as a locally closed subscheme) in HILB sending a triple of distinct points $a, b, c$ to the point of HILB given by the three length one subschemes of $V$ with ideal sheaves

$$
m_{a}, m_{b}, m_{c}
$$

the three length two subschemes of $V$ with ideal sheaves

$$
m_{a} \cdot m_{b}, m_{a} \cdot m_{c}, m_{b} \cdot m_{a}
$$

and the length three subscheme with ideal sheaf

$$
m_{a} \cdot m_{b} \cdot m_{c} .
$$

(Here $M_{a}$ is the maximal ideal of $a$.) Le Barz considers $\widehat{H}(V)$ only for smooth $V$ but it will be useful for us to have it defined in general. He demonstrates this space is smooth when $V$ is and gives the following global description: Let

$$
I_{1}, I_{2}, I_{3} \subset \mathscr{O}_{\text {HILB } \times V}
$$

Received by the editors May 1, 1990 and, in revised form, September 4, 1990.

1980 Mathematics Subject Classification (1985 Revision). Primary 14M99.

During this research the author was supported by a Sloan foundation doctoral dissertation fellowship and by an N.S.F. Postdoctoral fellowship. 
be the ideal sheaves of the universal families of length one subschemes associated with the three factors of $\operatorname{Hilb}_{1}(V)$. Let $I_{12}, I_{23}, I_{13}$ be the ideal sheaves of the universal families of length 2 subschemes associated with the three factors of $\operatorname{Hilb}_{2}(V)$ and let $I_{123}$ be the ideal sheaf of the universal family of length 3 subschemes associated with $\mathrm{Hilb}_{3}(V)$. Le Barz identifies $\widehat{H}$ as the locus in

$$
\operatorname{Hilb}_{1}(V)^{\times 3} \times \operatorname{Hilb}_{2}(V)^{\times 3} \times \operatorname{Hilb}_{3}(V)
$$

over which the universal ideals satisfy the relations

$$
\begin{aligned}
& I_{i} \cdot I_{j} \subset I_{i j} \subset I_{i} \quad \text { for } i \neq j, \\
& I_{i} \cdot I_{j k} \subset I_{123} \subset I_{j k} \text { for } i, j, k \text { distinct. }
\end{aligned}
$$

He describes this locus in terms of local coordinates for HILB and uses his local presentation to check that $\widehat{H}$ is smooth.

In [F-C], Fulton remarks that Le Barz's calculations imply that $\widehat{H}$ represents the functor $h_{3}$ from schemes to sets, an $S$ valued point of which is a collection of ideals

$$
I_{1}, I_{2}, I_{3}, I_{12}, I_{13}, I_{23}, I_{123} \subset \mathscr{O}_{S \times V}
$$

defining flat families of lengths 1,2 and 3 and satisfying the incidence relations:

$$
\begin{aligned}
& I_{i} \cdot I_{j} \subset I_{i j} \subset I_{i} \text { for } i \neq j, \\
& I_{i} \cdot I_{j k} \subset I_{123} \subset I_{j k} \text { for } i, j, k \text { distinct. }
\end{aligned}
$$

In this paper we exploit this observation. We show by some simple general remarks that the above functor is represented by a closed subscheme of HILB which we call $\mathrm{H}_{3}(V)$. By a series of functorial arguments we exhibit, in the case that $V$ is a smooth variety, $H_{3}(V)$ as a composition of blowups of "known" smooth varieties along "known" smooth subvarieties. In particular we conclude that $H_{3}(V)$ is a smooth variety and hence necessarily equal to $\widehat{H}(V)$, since the "honest" locus naturally embeds as an open subset of $H_{3}(V)$.

The blowup description is as follows: Let $V_{2}$ be the blowup of $V \times V$ along the diagonal. Let $V_{3}$ be the blowup of $V_{2} \times_{V} V_{2}$ along the diagonal. $\left(V_{2}\right.$ and $V_{3}$ are the first and second iteration schemes of the map of $V$ to a point, see [K11]). Let $T$ denote the blow up of

$$
P\left(\mathscr{T}_{V}\right) \times_{V} P\left(\mathscr{T}_{V}\right)
$$

(here $\mathscr{T}_{V}$ is the tangent bundle of $V$ ) along the diagonal. There is a canonical embedding of $T$ in $V_{3}$ and we show that $H_{3}(V)$ is the blowup of $V_{3}$ along $T$. This description was obtained by different methods in [K1] for the case of $\mathbb{P}^{2}$.

In the particular case of $\mathbb{P}^{n}$ we compare $\widehat{H}\left(\mathbb{P}^{n}\right)$ to the Schubert space of triangles, $\mathbb{S}\left(\mathbb{P}^{n}\right)$, which can be described as the closure in

$$
P(\mathscr{U}) \times_{G_{2}} P(\mathscr{U}) \times_{G_{2}} P(\mathscr{U}) \times_{G_{2}} G_{2}(\mathscr{U}) \times_{G_{2}} G_{2}(\mathscr{U}) \times_{G_{2}} G_{2}(\mathscr{U}) \times_{G_{2}} G_{3}\left(\operatorname{sym}_{2}(U)\right) .
$$

(Here $G_{2}$ is the grassmannian of planes in $\mathbb{P}^{n}$ and $\mathscr{U}$ is the universal rank three bundle on $G_{2}$ ) of the locus of "honest" triangles" (see [F-C]). The honest triangles embed in this product by sending the triple $a, b, c$ to the point of the product given by the data (in the plane spanned by the triple): the three points, the three, lines passing through two of the three points, and the net of conics in the spanning plane which pass through all three points. In $\S 3$ we give 
a functorial description of $\mathbb{S}\left(\mathbb{P}^{n}\right)$ and use it to realize this space as a blowup of $\widehat{H}\left(\mathbb{P}^{n}\right)$ along a smooth subvariety, the locus of triangles in $\widehat{H}\left(\mathbb{P}^{n}\right)$ which lie on some line. The referee points out that Rossello has obtained similar results in his thesis (see $[R])$.

The symmetric group $S_{3}$ acts on $H_{3}(V)$. In $\S 4$ we show that the quotient is smooth, when $V$ is, and exhibit it as the blowup of $\operatorname{Hilb}_{3}(V)$ along $G_{2}\left(\mathscr{T}_{V}\right)$ (the grassmannian of two planes in the tangent bundle). The latter space embeds in $\mathrm{Hilb}_{3}(V)$ by sending a plane in the tangent space at a point to the corresponding amorphous length 3 subscheme (when $V$ is two dimensional this is the subscheme whose ideal sheaf is the square of the maximal ideal). This quotient is a natural parameterizing space for unordered triangles, the "right" space for many enumerative applications. In addition it could be used to study the cohomology (or Chow ring) of $\mathrm{Hilb}_{3}(V)$.

In [F-C], for a plane curve $\mathscr{C}$ the space $\widehat{H}(\mathscr{C})$ is studied in relation to the problem of how many triangles are simultaneously inscribed in one plane curve and circumscribed about a second (there $\widehat{H}(\mathscr{C})$ is denoted $V_{\mathscr{C}}$ ). In particular an expression for its class in the codimension three Chow group is given (in case $\mathscr{C}$ has ordinary nodes and cusps; the case of general reduced plane curves is considered in [K2]). The expression depends on the degree of the curve as well as the local nature of the singularities. In particular if $\mathscr{C}_{t}$ is a flat family of plane curves with $\mathscr{C}_{t}$ smooth for nonzero $t$ and with $\mathscr{C}_{0}$ singular then the family $\widehat{H}\left(\mathscr{C}_{t}\right)$ is not flat. We show in $\S 5$ that if $\mathscr{D} \hookrightarrow X \times V$ is flat family of reduced divisors in the smooth variety $V$ then the triangle spaces $H_{3}\left(\mathscr{D}_{x}\right)$ (as $x$ varies over $X$ ) form a flat family of codimension three Cohen Macaulay subschemes of $\widehat{H}(V)$. In particular our methods exhibit for a reduced divisor $D$ in $V$ the triangle space $H_{3}(D)$ as the zero scheme of a regular section of a rank three vector bundle on $H_{3}(V)$. An interesting corollary of our methods is that $H_{3}(D)$ is equal to $\widehat{H}(D)$ when $D$ is normal.

I wish to thank Bill Fulton for bringing Le Barz's space to my attention, and for encouragement in general. I also wish to thank Steve Kleiman for a number of helpful discussions, and for supplying me with a preprint to his paper [K12], from which I drew considerable insight. In particular it was from Proposition 3.1 of that work that I got the idea of introducing the auxiliary functor $h_{3}^{\prime}$ (used towards the end of $\S 2$ ) which considerably simplified my argument.

Remarks on notations, conventions and assumptions. All of the schemes considered are assumed to be equidimensional, noetherian, and defined over a fixed algebraically closed field $k$ (of arbitrary characteristic). Unless otherwise noted for any scheme $S$ when we indicate ideals

$$
I_{i}, I_{i j}, I_{123} \subset \mathscr{O}_{S \times V} \text { with } i, j \in\{1,2,3\}
$$

we implicitly assume that the corresponding subschemes are flat over $S$ with $I_{i}$ defining a family of length one subschemes, $I_{i j}$ a family of length 2 subschemes and $I_{123}$ a family of length 3 subschemes.

Unless otherwise noted, by an embedding we mean a closed embedding, and by a variety we mean an irreducible integral scheme.

On any grassmannian, $\mathscr{U}$ will indicate the universal subbundle. For any variety $X$ we will denote the universal length 3 family of subschemes of $X$ on $H_{3}(X)$ by $U_{123}$ (pulling back the universal family on $\mathrm{Hilb}_{3}(X)$ ). 
Given families defined by ideals

$$
I_{1}, I_{2} \subset I_{12} \subset \mathscr{O}_{S \times V}
$$

the sheaf $I_{1} / I_{12}$ is locally free of length one over $S$ and hence its annihilator in $\mathscr{O}_{S \times V}$ defines a section of $S$. Thus we may think of the sheaf as a line bundle on $S$. If in addition $I_{1} \cdot I_{2} \subset I_{12}$ then this annihilator is necessarily $I_{2}$. Similar remarks apply to sheaves such as $I_{12} / I_{123}$.

We will make use, without remark, of the following elementary classification of subschemes of length at most three in a smooth variety. Any subscheme of length 2, supported at a point, is contained scheme theoretically in a curve, smooth at the point, and hence as a subscheme of the curve is defined by the square of the maximal ideal. Any subscheme of length 3 supported at a point is contained in a surface smooth at the point. A length 3 subscheme of a smooth surface (supported at a point) is either contained in a curve, smooth at the point, in which case it is defined by the cube of the maximal ideal and the subscheme is said to be curvilinear, or it is defined by the square of the maximal ideal of the surface at the point. The reader will easily supply a proof of this classification, or may (at somewhat greater effort) read of it in [B].

\section{REPRESENTING THE FUNCTORS}

In order to show that various functors described in the paper are represented we will make use of the following lemma. Definitions and elementary properties of Fitting ideals may be found in [La].

Lemma 1.1. Let $F \hookrightarrow S \times V$ be a flat family of subschemes of $V$ of length $d$ and let $G \hookrightarrow S \times V$ be any other (not necessarily flat or finite) family of subschemes of $V$. Let $I_{F}$ and $I_{G}$ be the ideal sheaves of $F$ and $G$, and $p$ the projection of $S \times V$ to $S$ and let $W$ be the subscheme of $S$ defined by the Fitting ideal $F_{d-1}\left(p_{*}\left(\mathscr{O}_{S \times V} /\left(I_{F}+I_{G}\right)\right)\right)$. Then $W$ is the unique subscheme of $S$ with the properties:

(1) The subscheme

$$
W \times{ }_{S} F \hookrightarrow W \times V
$$

factors through the subscheme

$$
W \times{ }_{S} G \hookrightarrow W \times V .
$$

(2) If $X \rightarrow S$ is any morphism such that the subscheme

$$
X \times{ }_{S} F \hookrightarrow X \times V
$$

factors through the subscheme

$$
X \times_{S} G \hookrightarrow X \times V
$$

then $X \rightarrow S$ factors through $W$.

Proof. Uniqueness is clear and so it is enough to prove that $W$ has the required properties. Let $X \stackrel{g}{\longrightarrow} S$ be any morphism. Let $F^{\prime}$ and $G^{\prime}$ be the pull backs of $F$ and $G$ and let $\pi$ and $\pi^{\prime}$ be defined by the cartesian diagram

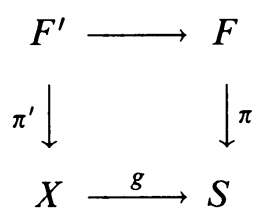


$F^{\prime}$ factors through $G^{\prime}$ if and only if the surjection

$$
\mathscr{O}_{F^{\prime}} \rightarrow \mathscr{O}_{X \times V} /\left(I_{F^{\prime}}+I_{G^{\prime}}\right) \rightarrow 0
$$

is an isomorphism. This holds (since $\pi^{\prime}$ is finite) if and only if the surjection

$$
\pi_{*}^{\prime}\left(\mathscr{O}_{F^{\prime}}\right) \rightarrow \pi_{*}^{\prime}\left(\mathscr{O}_{X \times V} /\left(I_{F^{\prime}}+I_{G^{\prime}}\right)\right) \rightarrow 0
$$

is an isomorphism. But since $\pi$ is finite this is the pull back

$$
g^{*} \pi_{*}\left(\mathscr{O}_{F}\right) \rightarrow g^{*} \pi_{*}\left(\mathscr{O}_{X \times V} /\left(I_{F}+I_{G}\right)\right) \rightarrow 0
$$

which by the universal property of the Fitting ideal is an isomorphism if and only if $g$ factors through $W$.

Definition 1. The scheme $W$ of the lemma is denoted $\operatorname{Inc}_{S}(F, G)$; it is called "inclusion scheme of $F$ in $G$ over $S$ " or also "inclusion scheme of $I_{G}$ in $I_{F}$ over $S . "$

For example the lemma shows that $h_{3}$ (defined in the Introduction and again in $\S 2$ ) is represented by a subscheme $H_{3}$ of

$$
\operatorname{Hilb}_{1}(V)^{\times 3} \times \operatorname{Hilb}_{2}(V)^{\times 3} \times \operatorname{Hilb}_{3}(V)
$$

as follows: Each of the inclusion relations in the definition of $h_{3}$ (for example $I_{12} \cdots I_{3} \subset I_{123}$ ) define (by the lemma) universal subschemes of

$$
\operatorname{Hilb}_{1}(V)^{\times 3} \times \operatorname{Hilb}_{2}(V)^{\times 3} \times \operatorname{Hilb}_{3}(V) \text {. }
$$

$H_{3}$ is the scheme theoretic intersection of all of these subschemes. The lemma also shows that if $X$ is a subvariety of $Y$ then $H_{3}(X)$ is the subscheme of $H_{3}(Y)$ defined by the condition that $U_{123}$, the length 3 family over $H_{3}(Y)$, factor through

$$
H_{3}(Y) \times X \hookrightarrow H_{3}(Y) \times Y .
$$

Thus we have the inequality

$$
H_{3}(X)=\operatorname{Inc}_{H_{3}(Y)}\left(U_{123}, H_{3}(Y) \times X\right) .
$$

2. BLOWUP DESCRIPTION OF $H_{3}(V)$

Throughout this section $V$ is assumed to be a smooth variety of dimension $n$.

Definition 2. Let $h_{3}$ be the functor from schemes to sets an $S$ valued point of which consists of a collection of ideals

$$
I_{1}, I_{2}, I_{3}, I_{12}, I_{23}, I_{13}, I_{123} \subset \mathscr{O}_{S \times V}
$$

satisfying the incidence relations

$$
\begin{aligned}
& I_{i} \cdot I_{j} \subset I_{i j} \subset I_{i} \text { for } i \neq j, \\
& I_{i} \cdot I_{j k} \subset I_{123} \subset I_{j k} \text { for } i, j, k \text { distinct. }
\end{aligned}
$$

Definition 3. Let $h_{2}$ be the functor, an $S$ valued of point of which consists of ideals

$$
I_{1}, I_{2}, I_{3}, I_{12}, I_{23}, I_{123} \subset \mathscr{O}_{S \times V}
$$


satisfying incidence relations

$$
\begin{aligned}
& I_{i} \cdot I_{j} \subset I_{i j} \subset I_{i} \text { for } i \neq j, \\
& I_{i} \cdot I_{j k} \subset I_{123} \subset I_{j k} \text { for } i, j, k \text { distinct } i \neq 2 .
\end{aligned}
$$

Definition 4. Let $h_{1}$ be the functor, an $S$ valued point of which consists of ideals

$$
I_{1}, I_{2}, I_{3}, I_{12}, I_{23} \subset \mathscr{O}_{S \times V}
$$

satisfying the incidence relations

$$
I_{i} \cdot I_{j} \subset I_{i j} \subset I_{i} \quad \text { for } i \neq j .
$$

Definition 5. Let $h_{0}$ be the functor, an $S$ valued point of which consists of ideals

$$
I_{1}, I_{2}, I_{3} \subset \mathscr{O}_{S \times V}
$$

Of course $h_{0}$ is represented by $V \times V \times V$.

Definition 6. Let $r$ be the functor, an $S$ valued point of which consists of ideals

$$
I_{1}, I_{2}, I_{12} \subset \mathscr{O}_{S \times V}
$$

satisfying the incidence relations

$$
I_{1} \cdot I_{2} \subset I_{12} \subset I_{1} \cap I_{2} \text {. }
$$

Lemma 1.1 shows that these are represented by subschemes

$$
\begin{aligned}
& H_{3} \hookrightarrow \operatorname{Hilb}_{1}(V)^{\times 3} \times \operatorname{Hilb}_{2}(V)^{\times 3} \times \operatorname{Hilb}_{3}(V), \\
& H_{2} \hookrightarrow \operatorname{Hilb}_{1}(V)^{\times 3} \times \operatorname{Hilb}_{2}(V)^{\times 2} \times \operatorname{Hilb}_{3}(V), \\
& H_{1} \hookrightarrow \operatorname{Hilb}_{1}(V)^{\times 3} \times \operatorname{Hilb}_{2}(V)^{\times 2}, \\
& H_{0}=\operatorname{Hilb}_{1}(V)^{\times 3}, \\
& R \hookrightarrow \operatorname{Hilb}_{1}(V)^{\times 2} \times \operatorname{Hilb}_{2}(V) .
\end{aligned}
$$

We have obvious maps of functors $h_{3} \rightarrow h_{2} \rightarrow h_{1} \rightarrow h_{0}$ inducing morphism of schemes $\mathrm{H}_{3} \rightarrow \mathrm{H}_{2} \rightarrow \mathrm{H}_{1} \rightarrow \mathrm{H}_{0}$.

We will show that $R \rightarrow V \times V$ realizes $R$ as the blowup along the diagonal, i.e. in the language of the introduction, $R$ is isomorphic to $V_{2}$. Since by definition $H_{1}$ is isomorphic to $R \times_{V} R$ this will imply that $H_{1}$ is isomorphic to $V_{2} \times V_{V}$.

Definition 7. Let $e_{13}$ be the functor, an $S$ valued point of which consists of ideals $I_{1}, I_{2}, I_{12} \subset \mathscr{O}_{S \times V}$ satisfying the incidence relations

$$
I_{1} \cdot I_{2} \subset I_{12} \subset I_{1} \cap I_{2} \text {. }
$$

By Lemma $1.1, e_{13}$ is represented by a subscheme $E_{13} \hookrightarrow H_{1}$ (our notation is chosen to comply with that of Le Barz) and this embedding represents the inclusion of functors $e_{13} \hookrightarrow H_{1}$ defined by sending

$$
I_{1}, I_{2}, I_{12} \rightarrow I_{1}, I_{2}, I_{1}, I_{12}, I_{12} \text {. }
$$

This realizes $E_{13}$ as the universal closed subscheme defined by the conditions $I_{1}=I_{3}$ and $I_{12}=I_{23}$. Observe that the first condition follows from the second, 
since the second implies that the line bundles $I_{2} / I_{23}$ and $I_{2} / I_{12}$ are equal, and their annihilators are $I_{3}$ and $I_{1}$ respectively. Under the isomorphism of $H_{1}$ with $R \times_{V} R, E_{13}$ corresponds to the diagonal. We show that $H_{2} \rightarrow H_{1}$ realizes $H_{2}$ as the blowup of $H_{1}$ along $E_{13}$, i.e. in the language of the introduction that the map gives an isomorphism of $\mathrm{H}_{2}$ with $V_{3}$.

Definition 8. Let $t$ be the functor, an $S$ valued point of which consists of ideals

$$
I_{1}, I_{12}, I_{23}, I_{123} \subset \mathscr{O}_{S \times V}
$$

satisfying the incidence relations

$$
I_{1}^{2} \subset I_{123} \subset I_{i j} \subset I_{1}
$$

By Lemma 1.1, $t$ is represented by a subscheme $T$ of $H_{2}$ and the embedding $T \hookrightarrow H_{2}$ represents the inclusion of functors $t \hookrightarrow h_{2}$ defined by

$$
I_{1}, I_{12}, I_{23}, I_{123} \rightarrow I_{1}, I_{1}, I_{1}, I_{12}, I_{23}, I_{123} \text {. }
$$

We will show that $T$ is isomorphic to the blowup of $P\left(\mathscr{T}_{V}\right) \times_{V} P\left(\mathscr{T}_{V}\right)$ along the diagonal and that $\mathrm{H}_{3} \rightarrow \mathrm{H}_{2}$ realizes $\mathrm{H}_{3}$ as the blowup of $\mathrm{H}_{2}$ along $T$.

The above description is obtained by a series of lemmas.

Lemma 2.1. The maps

$$
H_{3} \rightarrow H_{2}, \quad H_{2} \rightarrow H_{1}, \quad R \rightarrow V \times V
$$

are all proper.

Proof. We will consider $H_{3} \rightarrow H_{2}$. The proofs for the other maps are analogous.

We use the valuative criterion for properness. Let $\mathscr{C}$ be a smooth curve and $p \in \mathscr{C}$ a closed point. Let $U$ be the complement of $p$ in $\mathscr{C}$. Let

$$
I_{1}, I_{2}, I_{3}, I_{12}, I_{13}, I_{23}, I_{123} \subset \mathscr{O}_{U \times V}
$$

define a $U$ valued point of $H_{3}$ and let

$$
I_{1}^{\prime}, I_{2}^{\prime}, I_{3}^{\prime}, I_{12}^{\prime}, I_{23}^{\prime}, I_{123}^{\prime} \subset \mathscr{O}_{\mathscr{C} \times V}
$$

define a $\mathscr{C}$ valued point of $H_{2}$ such that the diagram

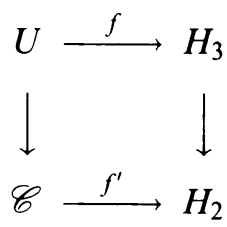

commutes. We show that $f^{\prime}$ lifts to an extension of $f$. Thus we seek an ideal $I_{13}^{\prime} \subset \mathscr{O}_{\mathscr{C} \times V}$ defining a flat family of length 2 subschemes, extending $I_{13}$ and satisfying

$$
I_{1}^{\prime} \cdot I_{3}^{\prime} \subset I_{13}^{\prime} \subset I_{1}^{\prime} \cap I_{2}^{\prime} \text {. }
$$

Let $Z \hookrightarrow \mathscr{C} \times V$ be the subscheme defined by the ideal $I_{1}^{\prime} \cdot I_{3}^{\prime} \subset \mathscr{O}_{\mathscr{C} \times V}$. Since $Z \rightarrow \mathscr{C}$ is finite it follows by [H, Proposition 9.8] that there exists an ideal $I_{13}^{\prime} \subset \mathscr{O}_{Z}$ defining a flat family of length 2 subschemes extending $I_{13}$. By Lemma 1.1, $I_{13}^{\prime}$ necessarily satisfies the above "incidence" conditions. This completes the proof. 
Lemma 2.2. $R \stackrel{p}{\longrightarrow} V \times V$ is an isomorphism away from the diagonal.

Proof. The map is birational as it is an isomorphism on the "honest" locus, i.e. on the open set where $I_{1}$ is not equal to $I_{2}$. Since the map

$$
R \backslash p^{-1}(\Delta) \rightarrow V \times V \backslash \Delta
$$

is proper and $V \times V$ is smooth it is enough to show that $p$ is one to one as a map of $k$-points away from $p^{-1}(\Delta)$. (For then the map is finite and birational.) To this end suppose $I_{1}, I_{2}, I_{12}$ and $I_{1}, I_{2}, I_{12}^{\prime}$ define distinct $k$-points of $R$. Then $I_{1}$ and $I_{2}$ are (by length considerations) both equal to $I_{12}+I_{12}^{\prime}$, i.e. this $k$-point is sent into the diagonal.

Lemma 2.3. The inverse image of the diagonal in $R$ is isomorphic to $P\left(\mathscr{T}_{V}\right)$.

Proof. The inverse image of the diagonal represents the functor, an $S$ valued point of which consists of ideals $I, J \subset \mathscr{O}_{S \times V}$ where $I$ defines a family of length 1 subschemes and $J$ defines a family of length 2 subschemes and such that

$$
I^{2} \subset J \subset I .
$$

Given an $S$ point of this functor, the ideal $I$ defines a section of $S \times V$ and hence a map $S \stackrel{f}{\longrightarrow} V$. The bundle $f^{*}\left(\Omega_{V}\right)$ is isomorphic to $I / I^{2}$ and $I / J$ is a line bundle quotient. Conversely given a map $S \stackrel{f}{\longrightarrow} V$ and a line bundle quotient $f^{*}\left(\Omega_{V}\right) \rightarrow \mathscr{L}$, let $I$ be the ideal sheaf of the graph of $f$, so that $f^{*}\left(\Omega_{V}\right)$ is isomorphic to $I / I^{2}$. Then the kernel of the surjection $I / I^{2} \rightarrow \mathscr{L}$ is of form $J / I^{2}$ for an ideal $J$ defining a flat family of length 2 . This data constitutes a point of the functor. This sets up an isomorphism of the inverse image of the diagonal with $P\left(\mathscr{T}_{V}\right)$.

We say that a subscheme is locally principal if its ideal sheaf is locally generated by a single element (which might be a zero divisor).

Lemma 2.4. The inverse image of the diagonal in $R$ is locally principal.

Proof. The inverse image of the diagonal is the zero scheme of the map of line bundles on $R$ which is the composition

$$
I_{1} / I_{12} \subset \mathscr{O}_{R \times V} / I_{12} \rightarrow \mathscr{O}_{R \times V} / I_{2} .
$$

Thus it is a locally principal subscheme.

From Lemmas 2.2-2.4 it follows that $R$ is smooth and irreducible, and the inverse image of the diagonal is a Cartier divisor. Thus $R \rightarrow V \times V$ factors through $V_{2}$. By Lemmas 2.2 and 2.3 the factored map can have no exceptional divisors and hence is an isomorphism. It follows that $H_{1}$ is isomorphic to $V_{2} \times V_{2}$.

Lemma 2.5. The map $H_{2} \rightarrow H_{1}$ is an isomorphism away from the inverse image of $E_{13}$.

Proof. The map is birational, an isomorphism on the "honest" locus, where the three ideals $I_{1}, I_{2}, I_{3}$ are distinct. Since the map is proper and $H_{1}$ is smooth it is enough to show that $H_{2} \rightarrow H_{1}$ is one to one (as a map of $k$-points) away 
from the inverse image of $E_{13}$. Suppose that

$$
\begin{gathered}
I_{1}, I_{2}, I_{3}, I_{12}, I_{23}, I_{123}, \\
I_{1}, I_{2}, I_{3}, I_{12}, I_{23}, I_{123}^{\prime}
\end{gathered}
$$

define $k$-points with the same image and assume that $I_{123} \neq I_{123}^{\prime}$. Since

$$
I_{123}+I_{123}^{\prime} \subset I_{12} \cap I_{23}
$$

this implies (by considering lengths) that $I_{12}$ and $I_{23}$ are equal and so the two points map into $E_{13}$.

Lemma 2.6. The inverse image of $E_{13}$ is a locally principal subscheme of $H_{2}$ and smooth and irreducible of dimension $3 n-1$.

Proof. The inverse image represents the functor, an $S$ valued point of which consists of ideals

$$
I_{1}, I_{2}, I_{12}, I_{123} \subset \mathscr{O}_{S \times V}
$$

satisfying the incidence relations

$$
I_{1} \cdot I_{2} \subset I_{12} \subset I_{1} \cap I_{2}, \quad I_{1} \cdot I_{12} \subset I_{123} \subset I_{12} .
$$

This functor is represented by $\mathbb{P}\left(I_{12} / I_{1} \cdot I_{12}\right)$ over $E_{13}$. We show that the sheaf $I_{12} / I_{1} \cdot I_{12}$ is locally free of rank $n$ by checking its dimension at each stalk. On the open set where $I_{1}$ is not equal to $I_{2}$ the sheaf is isomorphic to $I_{1} / I_{1}^{2}$ which is locally free of rank $n$. At a point where $I_{1}$ and $I_{2}$ are equal we may assume that in local coordinates $I_{1}$ is $\left(x_{1}, x_{2}, \ldots, x_{n}\right)$ and that $I_{2}$ is $\left(x_{1}^{2}, x_{2}, \ldots, x_{n}\right)$. One checks immediately that the dimension of $I_{12} / I_{1} \cdot I_{12}$ is $n$.

The inverse image is defined by the condition that $I_{12}=I_{23}$. Thus it is the zero scheme of the map of line bundles

$$
I_{12} / I_{123} \rightarrow I_{2} / I_{23}
$$

and hence locally principal.

As above these three results imply that the map $H_{2} \rightarrow H_{1}$ lifts to an isomorphism of $\mathrm{H}_{2}$ with $V_{3}$ and in particular imply that $\mathrm{H}_{2}$ is smooth and irreducible of dimension $3 n$.

Lemma 2.7. $t$ is isomorphic to the functor, an $S$ valued point of which consists of a map $S \stackrel{f}{\longrightarrow} V$ together with two sub line bundles and a rank two subbundle of $f^{*}\left(\mathscr{T}_{V}\right)$, such that each of the line bundles is a subbundle of the rank two bundle.

The proof is analogous to that of Lemma 2.3 and is omitted.

From the lemma it is clear that $T$ is isomorphic to the incidence variety in $G_{2}\left(\mathscr{T}_{V}\right) \times_{V} P\left(\mathscr{T}_{V}\right) \times_{V} P\left(\mathscr{T}_{V}\right)$ consisting of triples: a plane and two lines, with the lines contained in the plane. This subvariety is isomorphic to the blowup of $P\left(\mathscr{T}_{V}\right) \times_{V} P\left(\mathscr{T}_{V}\right)$ along the diagonal.

We now show that the map $\mathrm{H}_{3} \rightarrow \mathrm{H}_{2}$ realizes $\mathrm{H}_{3}$ as the blowup of $\mathrm{H}_{2}$ along $T$. We will make use of the following auxiliary functor. Let $h_{3}^{\prime}$ be the functor from schemes to sets an $S$ valued point of which consists of a collection 
of ideals $I_{1}, I_{2}, I_{3}, I_{12}, I_{23}, I_{123} \subset \mathscr{O}_{S \times V}$ defining an $S$ valued point of $H_{2}$ together with an ideal $I_{13} \subset \mathscr{O}_{S \times V}$ satisfying the incidence relations

$$
I_{123} \subset I_{13} \subset I_{1} \text {. }
$$

$h_{3}^{\prime}$ is represented by a closed subscheme

$$
H_{3}^{\prime} \hookrightarrow \operatorname{Hilb}_{1}(V)^{\times 3} \times \operatorname{Hilb}_{2}(V)^{\times 3} \times \operatorname{Hilb}_{3}(V) .
$$

There is a natural embedding $H_{3} \hookrightarrow H_{3}^{\prime}$ realizing $H_{3}$ as the subscheme defined by the conditions $I_{13} \subset I_{3}$ and $I_{2} \cdot I_{13} \subset I_{123}$.

Lemma 2.8. This embedding is an isomorphism on $k$-points.

Let

$$
I_{1}, I_{2}, I_{3}, I_{12}, I_{13}, I_{23}, I_{123} \subset \mathscr{O}_{V}
$$

define a $k$-point of $\mathrm{H}_{3}^{\prime}$. We show that this defines a point of $\mathrm{H}_{3}$ by showing that $I_{13} \subset I_{3}$ and that $I_{2} \cdot I_{13} \subset I_{123}$. For the first, either $I_{13}=I_{1} \cdot I_{3}$ or $I_{1}=I_{3}$. In either case $I_{13} \subset I_{3}$. For the second, $I_{13} / I_{123}$ is a one dimensional vector space supported at the point whose maximal ideal is $I_{2}$ and hence is annihilated by $I_{2}$. Thus $I_{2} \cdot I_{13} \subset I_{123}$.

We next show that $H_{3}^{\prime} \rightarrow H_{2}$ realizes $H_{3}^{\prime}$ as the blowup of $H_{2}$ along $T$. This will show in particular that $H_{3}^{\prime}$ is smooth and irreducible, and hence, by the above lemma necessarily equal to $\mathrm{H}_{3}$. We will make use of the following results:

Lemma 2.9. Given an exact sequence of modules

$$
L \rightarrow E \rightarrow M \rightarrow 0
$$

with $L$ and $E$ locally free of ranks 1 and 2 respectively there is an embedding of $\mathbb{P}\left(F_{1}(M)\right)$ in $\mathbb{P}(M)$ and this is an isomorphism when the Fitting ideal $F_{1}(M)$ is generated by a length 2 regular sequence.

Proof. The canonical map

$$
E \rightarrow \bigwedge^{2} E \otimes L^{*}
$$

factors through $M$. It has image $F_{1}(M) \otimes \bigwedge^{2} E \otimes L^{*}$ and has kernel $L$ (by the Kozul complex) in case $L$ in $E$ is given locally by a regular sequence of length 2 (which is equivalent to the given condition on $F_{1}$ ).

Observe that by the very definition $H_{3}^{\prime}$ is isomorphic to

$$
\mathbb{P}\left(I_{1} /\left(I_{1} \cdot I_{3}+I_{123}\right)\right)
$$

over $\mathrm{H}_{2}$. We have an exact sequence

$$
\left(I_{1} \cdot I_{3}+I_{123}\right) / I_{123} \rightarrow I_{1} / I_{123} \rightarrow I_{1} /\left(I_{1} \cdot I_{3}+I_{123}\right) \rightarrow 0
$$

Claim. The term on the left has stalks of dimension at most one.

Proof of Claim. We compute the dimension of the stalk at a $k$-point of $\mathrm{H}_{2}$. We consider only the case when the three ideals $I_{1}, I_{2}, I_{3}$ are equal. If $I_{123}$ is contained in $I_{1} \cdot I_{3}$ then of course the stalk is zero. Otherwise $I_{123}$ is curvilinear, and hence we may choose local coordinates $x_{1}, x_{2}, \ldots, x_{n}$ for $V$ so that $I_{123}$ is the ideal $\left(x_{1}^{3}, x_{2}, \ldots, x_{n}\right)$. One now checks immediately that the stalk is one dimensional.

By the above exact sequence the first Fitting ideal $F_{1}\left(I_{1} /\left(I_{1} \cdot I_{3}+I_{123}\right)\right)$ is equal to $F_{2}\left(\mathscr{O} /\left(I_{1} \cdot I_{3}+I_{123}\right)\right)$ which by Lemma 1.1 is the ideal sheaf of the inclusion scheme $I_{1} \cdot I_{3} \subset I_{123}$ (see Definition 1 in $\S 1$ ). 
Lemma 2.10. The inclusion scheme of $I_{1} \cdot I_{3}$ in $I_{123}$ (see Definition 1) is $T$. Proof. Let

$$
I_{1}, I_{2}, I_{3}, I_{12}, I_{13}, I_{23}, I_{123} \subset \mathscr{O}_{S \times V}
$$

define an $S$ valued point of this inclusion scheme. We show that $I_{1}=I_{2}=I_{3}$ and hence that it defines a point of $T . I_{2}$ is the annihilator in $\mathscr{O}_{S \times V}$ of $I_{3} / I_{23}$. One has

$$
I_{1} \cdot I_{3} \subset I_{123} \subset I_{23}
$$

thus $I_{1}$ is contained in $I_{2}$ and so is necessarily equal to $I_{2}$. Similarly $I_{3}=I_{2}$, which completes the proof.

Since $T$ is smooth and of codimension two in $\mathrm{H}_{2}$ it follows that the Fitting ideal $F_{1}\left(I_{1} /\left(I_{1} \cdot I_{3}+I_{123}\right)\right)$ is generated by a length 2 regular sequence and thus that $H_{3}^{\prime}$ is equal to the blowup of $H_{2}$ along $T_{2}$.

Remark. We have shown that $H_{3}(V)$ and $H_{3}^{\prime}(V)$ are equal when $V$ is smooth and irreducible. This implies that we have equality for arbitrary $V$.

Proof of Remark. Let $X$ be any scheme, the question is local, so we may assume $X$ embeds in a smooth irreducible $V$. Then we have a fibre diagram (see the remarks at the end of $\S 1$ )

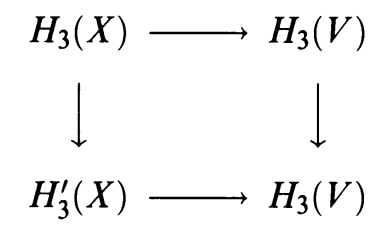

and the results follows.

\section{COMParison with SChUbert SPACE}

Throughout the section let $\mathbb{V}$ be a fixed $n+1$ dimensional vector space. We identify $\mathbb{P}^{n}$ with $P(\mathbb{V})$.

Let $s$ be the functor, an $S$ valued point of which consists of ideals

$$
I_{1}, I_{2}, I_{3}, I_{12}, I_{13}, I_{23}, I_{123} \subset \mathscr{O}_{S \times V}
$$

defining an $S$ valued point of $H_{3}\left(\mathbb{P}^{n}\right)$ together with a rank 3 subbundle

$$
E \subset \mathbb{V} \otimes \mathscr{O}_{S}
$$

such that the ideal of the corresponding projective space bundle, $P(E) \subset S \times \mathbb{P}^{n}$, satisfies $I_{P(E)} \subset I_{123}$.

By Lemma $1.1 \mathrm{~s}$ is represented by a closed subscheme

$$
\widehat{S} \hookrightarrow H_{3} \times G_{2}
$$

where $G_{2}=G_{2}\left(\mathbb{P}^{n}\right)$. The scheme $\widehat{S}$ is the inclusion scheme (Definition 1)

$$
\operatorname{Inc}_{H_{3} \times G_{2}}\left(U_{123} \times G_{2}, H_{3} \times P(\mathscr{U})\right) \text {. }
$$

Theorem 3.1. $\widehat{S}$ is isomorphic to $\mathbb{S}$, the Schubert space of triangles in $\mathbb{P}^{n}$.

Proof. Since the formation of the inclusion scheme commutes with all pullbacks, the fibres of the projection $\widehat{S} \rightarrow G_{2}$ are isomorphic to $H_{3}\left(\mathbb{P}^{2}\right)$ and thus in particular $\widehat{S}$ is smooth, connected, and proper of dimension $3 n$. 
We define a map $\widehat{S} \rightarrow \mathbb{S}$ as follows: Let $E \subset \mathbb{V} \otimes \mathscr{O}_{\widehat{S}}$ be the universal rank 3 bundle on $\widehat{S}$. We have surjections of sheaves on $\widehat{S} \times \mathbb{P}^{n}$

$$
\begin{gathered}
\mathscr{O}_{\widehat{S} \times \mathbb{P}^{n}}(1) \rightarrow \mathscr{O}_{P(E)}(1) \rightarrow\left(\mathscr{O} / I_{i}\right)(1), \\
\mathscr{O}_{\widehat{S} \times \mathbb{P}^{n}}(1) \rightarrow \mathscr{O}_{P(E)}(1) \rightarrow\left(\mathscr{O} / I_{i j}\right)(1), \\
\mathscr{O}_{P(E)}(2) \rightarrow\left(\mathscr{O} / I_{123}\right)(2) .
\end{gathered}
$$

We claim that these remain surjections after applying $p_{*}$, where $p$ is the projection onto $\widehat{S}$, and hence yield subbundles

$$
\begin{gathered}
p_{*}\left(\left(\mathscr{O} / I_{i}\right)(1)\right)^{*} \subset E \subset \mathbb{V} \otimes \mathscr{O}_{\widehat{S}}, \\
p_{*}\left(\left(\mathscr{O} / I_{i j}\right)(1)\right)^{*} \subset E \subset \mathbb{V} \otimes \mathscr{O}_{\widehat{S}}, \\
p_{*}\left(\left(\mathscr{O} / I_{123}\right)(2)\right)^{*} \subset \operatorname{sym}_{2}(E)
\end{gathered}
$$

on $\widehat{S}$. We will show that the map

$$
\operatorname{sym}_{2}\left(E^{*}\right) \rightarrow p_{*}\left(\left(\mathscr{O} / I_{123}\right)(2)\right)
$$

is a surjection, the other maps are handled similarly. By familiar base change theorems it is enough to establish the following:

Claim. If $Z$ is a length 3 subscheme of $\mathbb{P}^{2}$ then the map $\Gamma\left(\mathscr{O}_{\mathbb{P}^{2}}(2)\right) \rightarrow \Gamma\left(\mathscr{O}_{Z}\right)$ is surjective.

Proof of Claim. If $Z$ is not the subscheme of a line, then $\Gamma\left(\mathscr{O}_{Z}(1)\right)$ is zero, and thus the map $\Gamma\left(\mathscr{O}_{\mathrm{P}^{2}}(1)\right) \rightarrow \Gamma\left(\mathscr{O}_{Z}\right)$ is surjective (its kernel is zero, and both terms are three dimensional vector spaces) which implies the result. Thus we may assume $Z$ is a subscheme of $\mathbb{P}^{1}$ and it is enough to show that $\Gamma\left(\mathscr{O}_{\mathbb{P}}(2)\right) \rightarrow$ $\Gamma\left(\mathscr{O}_{Z}\right)$ is surjective. The map is injective ( $Z$ cannot be a subscheme of a point) and so is surjective since both terms are three dimensional.

These subbundles induce a map

$\widehat{S} \rightarrow P(\mathscr{U}) \times_{G_{2}} P(\mathscr{U}) \times_{G_{2}} P(\mathscr{U}) \times_{G_{2}} G_{2}(\mathscr{U}) \times_{G_{2}} G_{2}(\mathscr{U}) \times_{G_{2}} G_{2}(\mathscr{U}) \times_{G_{2}} G_{3}\left(\operatorname{sym}_{2}(U)\right)$ which clearly factors through $\mathbb{S}$.

We thus have a commutative diagram

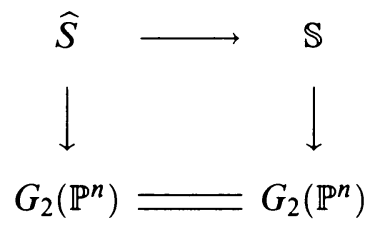

with the induced map between the fibres over a particular point of $G_{2}$ the constructed map $\widehat{S}\left(\mathbb{P}^{2}\right) \rightarrow \mathbb{S}\left(\mathbb{P}^{2}\right)$. Of course $\widehat{S}\left(\mathbb{P}^{2}\right)$ is equal to $H_{3}\left(\mathbb{P}^{2}\right)$. Le Barz remarks in [L] that this map is an isomorphism (both spaces are smooth and the map is proper and birational. On easily checks that the fibres are finite). This can also be seen from our blowup description for $H_{3}\left(\mathbb{P}^{2}\right)$ which agrees with the blowup description for $\mathbb{S}\left(\mathbb{P}^{2}\right)$ given in [K1]. In any case this shows that $\widehat{S}$ and $\mathbb{S}$ are isomorphic and completes the proof.

We now relate $\mathbb{S}\left(\mathbb{P}^{n}\right)$ to $H_{3}\left(\mathbb{P}^{n}\right)$. We have the projection $\mathbb{S} \stackrel{\pi}{\longrightarrow} H_{3}$ representing the forgetful functor. We will realize this as a blowup. 
Let $l$ be the functor, an $S$ valued point of which consists of ideals

$$
I_{1}, I_{2}, I_{3}, I_{12}, I_{23}, I_{123} \subset \mathscr{O}_{S \times V}
$$

defining an $S$ valued point of $H_{3}\left(\mathbb{P}^{n}\right)$ together with a rank 2 subbundle $F \subset$ $\mathbb{V} \otimes \mathscr{O}_{S}$ such that the ideal sheaf of the corresponding projective space bundle $P(F) \subset S \times \mathbb{P}^{n}$ satisfies $I_{P(F)} \subset I_{123}$.

By Lemma 1.1, $l$ is represented by a closed subscheme $L\left(\mathbb{P}^{n}\right)$ of $H_{3}\left(\mathbb{P}^{n}\right) \times$ $G_{1}\left(\mathbb{P}^{n}\right)$.

The fibres of $L \rightarrow G_{1}$ are isomorphic to $H_{3}\left(\mathbb{P}^{1}\right)$ and in particular $L$ is proper, connected and smooth of dimensions $2 n+1$.

Lemma 3.2. $L \rightarrow H_{3}$ is an embedding.

Proof. Since the map is proper it is sufficient to show that the corresponding map of functors is injective. As in the proof of Theorem 3.1 the composition of surjections on $L \times \mathbb{P}^{n}$,

$$
\mathscr{O}_{L \times \mathbb{P}^{n}}(1) \rightarrow \mathscr{O}_{P(F)}(1) \rightarrow\left(\mathscr{O} / I_{12}\right)(1)
$$

induces subbundles

$$
p_{*}\left(\left(\mathscr{O} / I_{12}\right)(1)\right)^{*} \subset F \subset \mathbb{V} \otimes \mathscr{O}_{L}
$$

( $p$ is the projection onto $L$ ). Since both are of rank 2 we conclude that

$$
p_{*}\left(\left(\mathscr{O} / I_{12}\right)(1)\right)^{*}=F \text {. }
$$

$L$ is the locus of triangles in $\widehat{H}\left(\mathbb{P}^{n}\right)$ which are contained (scheme theoretically) in some line.

Theorem 3.3. $\mathbb{S}$ is the blowup of $\mathrm{H}_{3}$ along $L$.

Proof. Let $\widehat{L}$ be the inverse image of $L$ under $\pi$. An $S$ valued point of $\widehat{L}$ consists of an $S$ valued point of $L$ given by ideals

$$
I_{1}, I_{2}, I_{3}, I_{12}, I_{23}, I_{123} \subset \mathscr{O}_{S \times V}
$$

and a rank 2 subbundle $F \subset \mathbb{V} \otimes \mathscr{O}_{S}$ plus a rank 3 subbundle $E \subset \mathbb{V} \otimes \mathscr{O}_{S}$ satisfying $I_{P(E)} \subset I_{123}$.

Claim. Necessarily $F$ is a subbundle of $E$.

Proof. In the proof of 3.2 we realized $F$ as $p_{*}\left(\left(\mathscr{O} / I_{12}\right)(1)\right)^{*}$ and in the proof of 3.1 we showed that this was a subbundle of $E$.

By the claim, $\widehat{L}$ is isomorphic to $\mathbb{P}(\mathbb{V} / F)$ and thus in particular a smooth irreducible Cartier divisor of $\mathbb{S}$.

Claim. $\mathbb{S} \backslash \widehat{L} \rightarrow H_{3} \backslash L$ is an isomorphism.

Proof. Let $W$ be the open set $\mathbb{S} \backslash \widehat{L}$. One shows as in the proof of Theorem 3.1 that the map

$$
p_{*}\left(\mathscr{O}_{P(E)}(1)\right) \rightarrow p_{*}\left(\left(\mathscr{O} / I_{123}\right)(1)\right)
$$

is surjective on $W$. This yields the equality of bundles on $W$,

$$
E=p_{*}\left(\left(\mathscr{O} / I_{123}\right)(1)\right)^{*}
$$

and implies the result.

It now follows that $\mathbb{S}$ is the blowup of $H_{3}$ along $L$. 


\section{A SPACE OF UNORDERED TRIANGLES}

Through this section we will work with a fixed smooth variety $V$ which we will often omit from the notation. The aim of this section is to prove

Theorem 4.1. The quotient of $\mathrm{H}_{3}$ by the symmetric group is isomorphic to the blowup of $\mathrm{Hilb}_{3}(V)$ along $G_{2}\left(\mathscr{T}_{V}\right)$.

Denote this quotient by $\widetilde{H}_{3}$. The natural map of $H_{3}$ to $\mathrm{Hilb}_{3}$ factors through $\widetilde{H}_{3}$. We embed $G_{2}\left(\mathscr{T}_{V}\right)$ in $\mathrm{Hilb}_{3}$ as follows: An $S$ valued point of $G_{2}$ is given by ideals $I, I_{123} \subset \mathscr{O}_{S \times V}$ defining families of length 1 and 3 and satisfying $I^{2} \subset I_{123} \subset I$.

(See the proof of Lemma 2.3.) Thus there is a natural map of $G_{2}$ to $\mathrm{Hilb}_{3}$.

Lemma 4.2. This map is an embedding.

Proof. The map clearly gives an embedding on closed points, and so it is enough to consider tangent vectors. A tangent vector to the product $\mathrm{Hilb}_{1} \times \mathrm{Hilb}_{3}$ at the point of $G_{2}$ given by ideals $I, I_{123} \subset \mathscr{O}_{V}$ consists of two maps

$$
I \stackrel{\phi}{\longrightarrow} \mathscr{O} / I, \quad I_{123} \stackrel{\phi_{123}}{\longrightarrow} \mathscr{O} / I_{123}
$$

(see [H, Exercise 9.7]).

Claim. This defines a tangent vector to $G_{2}$ if and only if the following two conditions are satisfied:

(1) The diagram

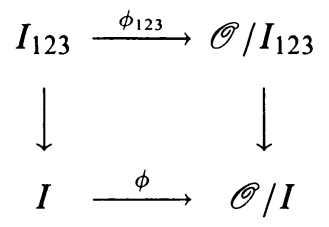

commutes. Here the vertical maps are the natural inclusion and surjection.

(2) $\phi_{123}(x \cdot y)=\phi(y)+y \cdot \phi(x) \bmod I_{123}$ for $x, y \in I$.

The above description of the tangent space is due to Bill Fulton.

Proof of Claim. We may assume that $V$ is $\operatorname{Spec}(A)$. Then the maps $\phi$ and $\phi_{123}$ induce families of subschemes of $V$ parameterized by the dual numbers with ideals

$$
J, J_{123} \subset A[\varepsilon] / \varepsilon^{2}
$$

defined as follows: $J$ is the set of elements of form

$$
x+\phi(x) \cdot \varepsilon \text { for } x \in I
$$

and $J_{123}$ is the set of elements of form

$$
z+\phi_{123}(z) \cdot \varepsilon \text { for } z \in I_{123}
$$

(again see [H, Exercise 9.7]). On easily checks that $J_{123}$ is contained in $J$ if and only if condition (1) above is satisfied, and that $J^{2}$ is contained in $J_{123}$ if and only if condition (2) is satisfied. This establishes the claim.

One may choose local coordinates $x_{1}, \ldots, x_{n}$ for $V$ so that

$$
I=\left(x_{1}, \ldots, x_{n}\right), \quad I_{123}=\left(x_{1}^{2}, x_{1} \cdot x_{2}, x_{2}^{2}, x_{3}, \ldots, x_{n}\right) \text {. }
$$


One checks easily that the conditions of the claim imply that $\phi_{123}$ determines $\phi$. Thus the map on tangent vectors is injective, which completes the proof.

Denote by $\widetilde{\mathrm{Hilb}_{3}}$ the blowup of $\mathrm{Hilb}_{3}$ along $G_{2}$. We show that

$$
\widetilde{H}_{3} \stackrel{f}{\longrightarrow} \mathrm{Hilb}_{3}
$$

factors through $\widetilde{\mathrm{Hilb}}_{3}$. For this (by the universal property of the quotient and of the blowup) it is enough to show that the inverse image of $G_{2}$ in $H_{3}$ is smooth of codimension 1 . An $S$ point of this inverse image is given by ideals

$$
I_{i}, I_{i j}, I_{123}, I \subset \mathscr{O}_{S \times V}
$$

where the $I_{i}, I_{i j}, I_{123}$ define families of length 1,2 and 3 and satisfy the usual relations, and $I$ defines a family of length 1 and satisfies the relation $I^{2} \subset$ $I_{123} \subset I$.

Lemma 4.3. The above functor is the same as the functor, an $S$ valued point of which consists of ideals

$$
I, I_{12}, I_{13}, I_{23}, I_{123} \subset S \times V
$$

satisfying the relations $I^{2} \subset I_{123} \subset I_{i j} \subset I$.

Proof. The two functors are easily seen to have the same set of closed points, and so it is enough to check that they yield the same tangent vectors. The argument is similar to that given in the lemma above and the reader will easily supply the details.

Observe that the functor of the lemma is represented by

$$
P(\mathscr{U}) \times_{G_{2}} P(\mathscr{U}) \times_{G_{2}} P(\mathscr{U})
$$

(see the proof of Lemma 2.3). In particular it follows that the inverse image of $G_{2}$ in $H_{3}$ is smooth and of codimension one, and that the inverse image in $\widetilde{H}_{3}$ (its quotient by $\left.S_{3}\right)$ is $\left.P\left(\operatorname{sym}_{3}\right)(\mathscr{U})\right)$. Thus $f$ factors to give a map

$$
\widetilde{H}_{3} \rightarrow \widetilde{\mathrm{Hilb}}_{3} \text {. }
$$

The map is clearly birational. It is proper by an argument similar to that of Lemma 2.1 and hence surjective. Also one checks easily that it is settheoretically finite away from the inverse images of $G_{2}$ (in fact set-theoretically finite away from the inverse images of $G_{2}$ (in fact set-theoretically an isomorphism). Finally, it is finite on the inverse image of $G_{2}$ since on fibers over $G_{2}$ it is a surjective map of $\mathbb{P}^{3}$ to itself. Thus it is finite and birational and hence, since the image is smooth, an isomorphism.

\section{TRIANGLES IN A FAMILY OF REDUCED DIVISORS}

Throughout this section $V$ denotes a smooth $n$ dimensional variety.

Let $\mathscr{D} \hookrightarrow X \times V$ be a flat family of reduced Cartier divisors parameterized by $X$. In this section we prove that the collection of triangle spaces $H_{3}\left(\mathscr{D}_{x}\right)$ (as $x$ runs over $X$ ) form a flat family of codimension three Cohen-Macaulay subschemes of $\mathrm{H}_{3}(V)$.

Form the inclusion scheme (Definition 1)

$$
\operatorname{Inc}_{H_{3}(V) \times X}\left(U_{123} \times X, H_{3}(V) \times \mathscr{D}\right) .
$$


We will refer to this space as $H_{3}(\mathscr{D} / X)$. It has a natural projection to $X$ and since the formation of the inclusion scheme commutes with all base extensions it follows that the fibre of this projection over a closed point $x$ is the subscheme $\operatorname{Inc}_{H_{3}(V)}\left(U_{123}, H_{3}(V) \times \mathscr{D}_{x}\right)$ of $H_{3}(V)$ which is isomorphic to $H_{3}\left(\mathscr{D}_{x}\right)$ (see remarks at the end of $\S 1)$. Our objective is to prove that $H_{3}(\mathscr{D} / X)$ is flat over $X$. We begin with a general observation.

Let $\mathscr{E}, F \hookrightarrow B \times V$ be two flat families of subschemes of $V$ parameterized by a scheme $B$. Assume $\mathscr{E}$ is a family of Cartier divisors and $F$ is a family of length $n$ subschemes. There is a canonical section

$$
\mathscr{O}_{F} \stackrel{\dot{s}}{\rightarrow} \mathscr{O}_{F} \otimes \mathscr{O}(\mathscr{E})
$$

which induces a section

$$
\mathscr{O}_{B} \stackrel{s}{\longrightarrow} \pi_{*}\left(\mathscr{O}_{F} \otimes \mathscr{O}(\mathscr{E})\right)
$$

where $\pi$ is the projection onto the first factor.

Lemma 5.1. The zero scheme of $s$ is the inclusion scheme $\operatorname{Inc}_{B}(F, \mathscr{E})$.

Proof. Let $Y \stackrel{f}{\longrightarrow} B$ be a morphism. Define $g$ and $\pi^{\prime}$ by the fibre diagram

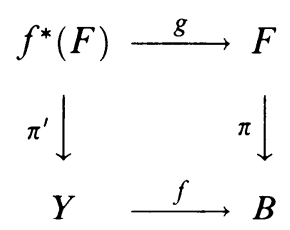

It is clear that $f^{*}(F)$ factors through $f^{*}(\mathscr{E})$ if and only if $g^{*}(\tilde{s})$ is zero. $g^{*}(\tilde{s})$ is zero if and only if $\pi_{*}^{\prime}\left(g^{*}(\tilde{s})\right)$ is zero. Since $\pi$ is finite

$$
\pi_{*}^{\prime}\left(g^{*}(\tilde{s})\right)=f^{*}\left(\pi_{*}(\tilde{s})\right)=f^{*}(s) .
$$

Thus $f^{*}(F)$ factors through $f^{*}(\mathscr{E})$ if and only if $f$ factors through the zero scheme of $s$.

By the lemma (with $B=H_{3}(V) \times X, F=U_{123} \times X$ and $\mathscr{E}=H_{3}(V) \times \mathscr{D}$ )

$$
H_{3}(\mathscr{D} / X) \hookrightarrow H_{3}(V) \times X
$$

is the zero scheme of the canonical section

$$
\mathscr{O}_{H_{3}(V) \times X} \stackrel{s}{\longrightarrow} \pi_{*}\left(\mathscr{O}_{U_{123} \times X} \otimes \mathscr{O}\left(H_{3}(V) \times \mathscr{D}\right)\right) .
$$

The zero scheme of the restriction of $s$ to the fibre over $x$ is then the fibre of $H_{3}(\mathscr{D} / X)$, which is $H_{3}\left(\mathscr{D}_{x}\right)$. We wish to show that $s$ is a regular section, that its restriction to each fibre is regular, and that its zero scheme is flat over $X$. By the local criterion of flatness (see [M, Corollary 1, p. 151]) it is enough to establish that the restriction of $s$ to each fibre is regular, and for this, since $H_{3}(V)$ is smooth, it is enough to count dimensions.

Lemma 5.2. If $D$ is a reduced Cartier divisor of a smooth $n$ dimensional variety $V$ then the dimension of $H_{3}(D)$ is at most $3 \cdot n-3$.

Proof. This is a simple dimension count. Let $C$ be an irreducible subvariety of $\mathrm{H}_{3}(D)$. We bound the dimension of $C$ by considering the various possibilities for the length 3 subscheme of its generic point. If it is supported at three 
distinct points, then $C$ has dimension at most $3 \cdot(n-1)$. If it is supported at two points of $D$, with the "double point" a smooth point of $D$ then $C$ has dimension at most $3 \cdot n-4$ (we have $2 \cdot n-2$ dimensions for the points, and $n-2$ dimensions for a tangent vector at the double point). If it is supported at two points, with the double point a singular point of $D$, then its dimension is at most $3 \cdot n-4$ (we have $n-2$ dimensions for the singular point, $n-1$ for the other point, and $n-1$ for a tangent vector at the singular point). If it is supported at one smooth point of $D$ then its dimension is at most $3 \cdot n-4$. Finally if it is supported at one singular point of $D$ then its dimension is at most $3 \cdot(n-1)$ because this is the dimension of the locus in $H_{3}(V)$ supported at singular points of $D$.

We have thus established

Theorem 5.3. If $\mathscr{D} \hookrightarrow X \times V$ is a flat family of reduced Cartier divisors then the family $H_{3}(\mathscr{D} / X) \hookrightarrow H_{3}(V) \times X$ is regularly embedded and flat over $X$. Its fibre over a point $x$ of $X$ is $H_{3}\left(\mathscr{D}_{x}\right)$ and each of these fibres is a regularly embedded codimension three subscheme of $\mathrm{H}_{3}(V)$.

Remark. By a similar dimension count as that used in the lemma, one can show that $H_{3}(D)$ is integral and hence equal to $\widehat{H}(D)$ when $D$ is normal.

\section{REFERENCES}

[B] J. Briancon, Description de Hilb ${ }^{n} \mathbb{C}\{x, y\}$, Invent. Math. 41 (1977), 45-89.

[F-C] W. Fulton and A. Collino, Intersection rings of spaces of triangles (preprint).

[H] R. Hartshorne, Algebraic geometry, Springer-Verlag, 1977.

[K1] S. Keel, Intersection theory of polygon spaces and incidence varieties, Comm. Algebra (to appear).

[K2] _ Triangles inscribed in reduced plane curves (unpublished).

[K11] S. Kleiman, Multiple point formulas 1: Method of iteration, Acta Math. 147 (1981).

[K12] _- Multiple point formulas 2: The Hilbert scheme (preprint).

[La] S. Lang, Algebra, Addison-Wesley, 1984.

[L] P. Le Barz, La variété des triplets completes, Duke Math. J. 57 (1988), 925-946.

[H] H. Matsummura, Commutative algebra, Benjamin/Cummings, 1980.

[R] F. Rossello, Calculs de grups de Chow i aplicacions, Thesis, University of Barcelona, Departament de Geometria i Topologia. 